\title{
Social Media in Contemporary China: A Transformative Public Sphere?
}

\author{
Bei Guo \\ School of Journalism and Communication, Shaanxi Normal University, No. 620 West Chang'an Street, \\ Chang'an District, Xi'an, Shaanxi Province, P.R.China \\ Email: bei.guo@snnu.edu.cn \\ *Corresponding author: Bei Guo
}

Keywords: Weibo, public sphere, public space

\begin{abstract}
Weibo is a Chinese invented social media platform integrating key features of Facebook and Twitter, which make it a seemingly freer channel for individual to individual communication, so bypassing the gaze of authority. It has been extolled by its supporters as offering a vital public sphere, as a space for the free exchange of public opinion. However, as a medium, it faces a number of significant limitations, which undermine its ability to offer a true "public sphere". First there is a clear imbalance in the demographics of Weibo users that prevents it from being truly representative of the Chinese people. Second, it is understood by scholars that a "public sphere" offers a forum for exchange of citizen's views on issues of the day based on a level of rationality. By comparison, Weibo is dominated by trivia, personal exposé and gossip. Finally and most importantly, Weibo does not evade the gaze of authority, which has simply adapted in order to monitor any postings on the site. Weibo may give the illusion of being a free speech platform that transforms China's public sphere, when in fact it is simply another public space for social communication within the permissible discourses of the state.
\end{abstract}

\section{Introduction}

In October 2010, a 22-year-old man named Li Qiming, driving a car, struck and killed one person and injured another on the campus of Hebei University. When arrested, Li Qiming yelled out to the angry crowd that had gathered, "Go ahead and sue me, my father is Li Gang”. Li Gang was the Deputy Director of the local police department. This event quickly stirred public outcry on China's most popular microblogging site, Weibo, with millions of online comments. "My father is Li Gang" quickly became a popular Internet expression referring to misdeeds of the privileged second generation children of officials, as China's netizens believed that the events reflected the arrogance of these offspring of powerful figures.

The past few years has witnessed many major events that were first exposed on Weibo. After a series of widespread and heated online discussions, an event will then become a virtual public event, which places intense pressure on the authorities and pushes the latter to respond through existing policies or practices. To some extent then, Weibo is able to alter the progress of the events. There are many examples of this, and a number of them concern illegal behaviour by privileged people, who in the past would have been protected from public exposure.

Weibo has been brought into Chinese society in 2008, though its history may be short, its spreading use is nonetheless breathtaking. Incidents such as the "Li Gang" event have led many scholars to believe that the development of the internet poses serious challenges to Chinese society (Yang, 2009; Zheng, 2008). Proponents of Weibo expect that online discourse will increase political participation and pave the way for democratic utopia. However, there is also scholarship expressing concern over an unqualified view of a democratising role for Weibo, as the Chinese authorities have evolved sophisticated strategies in their regulations of Weibo. Therefore, it is important to examine whether Weibo and its surrounding technologies will truly offer a political sphere or they are merely adapted to the current status quo. Since the concept of the public sphere has travelled widely and attracted much scholarly attention, this article attempts to propose its understandings shaped by 
local challenges. Through exploring several aspects that augment this potential, this study argues that Weibo is a social media platform which is primarily a public space for communication; however it is not a complete liberational tool, therefore cannot meet the definition of a fulfilled public sphere.

\section{The Public Sphere}

The term "public sphere" is usefully explored by Habermas, writing of its origins in $17^{\text {th }}$ and $18^{\text {th }}$ century Europe as a domain of social life in which public opinion could be formed (Habermas, 1991). These discussions can be generally distinct from and critical of the state. However, the public sphere declines because of the advent of modern mass media for its commercialization and the emergence of industrial society since the later part of the 19th century that stifle public input into debates. These conceptualizations are subject to some critics for their somewhat romanticisation. It is not equal to compare Kant in the $18^{\text {th }}$ century with suburban television viewers of the $20^{\text {th }}$ century (Calhoun, 1992). What is consistent though, is the theory that whether a public sphere is sufficient to create a democratic polity depends on the quality of its content and the degree to which citizens participate. A spirit of rationality in the discourse and its openness to popular participation are central (Calhoun, 1992, pp.2-4).

Both conceptualizations and critics of the public sphere focused on the 'physical space', which mentioned by Habermas as coffee house, salons and mass media. Verstraeten (1996) contends that since an ideal public sphere has never been fully established, it is necessary to actualize the concept in accordance with the social context. Moreover, Castells (2008) argues the concept of public sphere is in a changing process, and it varies in terms of context, history and technology. Therefore, Habermas's public sphere concept is constantly being re-examined in light of the rapid change in communication technologies in recent years. In the existing scholarship on the internet and public sphere, Yang (2009) applies the definition of the public sphere and focuses on "free spaces" where citizens could have public expression.

In these works, the internet is considered as a means to facilitate user-generated content, expanding the space for freedom of speech and providing a platform for public discussion, especially with the advent of social media, including personal blogs, Twitter and Weibo, where we have seen an explosion of debate. It now seems possible to simply idealize the concept of the public sphere as it occupies the internet, and to overlook that some sectors of society are routinely excluded and there is not a complete review of the public sphere in the Weibo of public space and public sphere. Whereas public space offers another forum for public discussion, public sphere promotes facilitates public discussion and enhances democracy. Therefore, a public space does not necessarily equal a democratic public sphere (Papacharissi 2002).

It is of great importance to study the potential of Weibo, in particular in the social media era, the power of the internet is even more powerful with the rise of social media. Weibo provides an opportunity for the netizens to skilfully make sophisticated critiques of the regime where Twitter has been successfully used to cause social mobilization such as Arab Spring uprisings. It is necessary to take a cautious approach to study Weibo's political impact under specific Chinese socio-cultural context.

\section{The Nature of Weibo}

Weibo has emerged as the most influential microblogging platform which combines the features of Twitter and Facebook, allowing "real-time" communications among large networks of individuals. It has developed rapidly as an information exchange platform following the creation of blogs, and has become an important forum for public opinion in China. Weibo's function is more informative and hence, more powerful than its Twitter counterpart. Besides, the unique 'forward' function of Weibo could enable an user to instantly reach hundreds or even thousands of "followers". The next few sections will make an illustration on several aspects of Weibo which curtail its potential. In order to assess Weibo's capacity to offer a meaningful public sphere while 
adequately reflecting China's specific social context, this study analyses Weibo from three key perspectives: first, its significant demographic imbalances in the user population which prevent it from truly representing Chinese citizens, second, the focus and quality of its general discourse, and third, the continued scrutiny of its content by various levels of authority. While these examples show the impact of Weibo, the next section lists a number of significant limitations that undermine Weibo's ability to offer a true public sphere. First, we examine the question of how accessible and representative Weibo is as a public medium.

\subsection{Demographics of Weibo}

The first lens through which Weibo must be examined as a potential public sphere, is the extent of its accessibility and representativeness, as this takes into account a detailed profiling of its users based on gender, age, education, and social status, mainly from an income perspective.

By the end of 2013, China has reached a total of 618 million internet users; with the internet penetration rate is $45.8 \%$. Meanwhile the number of Weibo users is 281 million, making up $44.5 \%$ of the country's total internet users. Although there is still no completely accurate number of regular users of Weibo, within the entire population of 1.4 billion, 281 million is a relatively small number and cannot be seen as representative of the whole population. This fact must be considered when drawing conclusions about the extent and impact of Weibo users in creating or contributing to a public sphere.

A closer demographic analysis of Weibo users shows that young people is the dominant group, with age group of 10-39 years accounting for $80 \%$. Furthermore, almost three quarters of Weibo users own lower than high school level of education. In addition, low income and no income groups are the majority, with the majority are students, supported by their parents. Therefore, the majority of microbloggers are not the middle class who are the backbone of the society. From a geographical perspective, it has been reported that Weibo users are mainly concentrated in the economically developed eastern regions such as Beijing and Shanghai as well as wealthier coastal regions. This geographical skewing of users towards the urban further reinforces Weibo's lack of representativeness of the whole nation.

In addition, less than $1 \%$ of Weibo users in the random sample are actively producing new content and engaging discussion, therefore, a significant portion of Weibo accounts are inactive in terms of contribution, and only a small group of microbloggers were responsible for creating a majority of content and for engaging the attention of other users (Fu \& Chau 2013). In this way, the numbers are not efficient and satisfactory to measure Weibo's political impact.

Moreover, the pursuit of democracy in China's new media landscape is the concern of "a tiny intellectual minority", as over two thirds of internet users in China concerns entertainment and consumerism" (Castells, 2009, p.285). The microbloggers tend to show more concern for everyday life matters such as food safety rather than state or international politics (Blue book of new media 2013). The fact that Weibo is only accessible to a small fraction of the population produces a virtual public space that does not in key respects meet the fundamental criteria of a public sphere.

\subsection{Internet Celebrities}

One of the main prerequisites of a Habermasian public sphere is that it is a forum for rational critical discussion among citizens about common concerns. The quality of participation emphasizes an informed, reasoned and interactive public debate. A public sphere can only be realized by rational-critical debate, potentially informing or evolving into mainstream public opinion (Habermas, 1991, p.219). By these measures, Weibo frequently undermines the functions of a public sphere, and there are regular striking examples of the misuses of Weibo as a public space. Tawdry stories of internet celebrities are common.

On August 20th, 2013, two influential web figures, Qin Huohuo (real name Qin Zhihui) and Lierchaisi (real name Yang Xiuyu) were detained by Beijing police on criminal charges. Initially they had spread a series of rumours on Weibo to attract attention and build an increasing number of followers. In March 2010, Qin and Yang launched a lucrative business and opened a company although for which no website could be found. This was a Chinese "water army" (shuijun) company 
paid by other companies to artificially generate grassroots online activity for their benefit. Their client services included web marketing, creating scandals or events and deleting negative comments from online forums. All these efforts had a very simple purpose: to make them famous.

These cases show that Weibo is occasionally simply used as a platform by internet celebrities to fabricate false information. Many of internet celebrities have millions of followers and some write provocatively about sensitive social and political issues to make internet incidents controversial, thus influencing the ideas of the followers and the direction of certain events. This is an issue surely of great concern to the authorities as it can easily spread rumour as well as factual information.

While a large number of users often participate in discussions on Weibo, it is not only the participation per se which is of critical importance, but also the content of the discussion and the quality of the discourse that plays an indispensable part in constituting a public sphere (Calhoun, 1992, pp.2-4). Though Weibo has altered traditional agenda-setting, the increasing numbers of users and the rise of public opinion expressed do not necessarily equate to the rational discussion in the public sphere. Moreover, the emergence of opinion leaders on Weibo does not necessarily promote social solidarity; it is easier to create a high level of societal fragmentation because people are more likely to move in the same direction as their own groups. This concern was also raised by Habermas (2009, p.53) by saying that the horizontal communication of the internet has also diminished the role of intellectuals in the traditional public sphere.

Weibo has also been strongly criticised for its 'human flesh search engine' (HFSE) or 'Renrou (human flesh) search engine', which is used specifically by the Chinese media for online searches for people, with the aim of discrediting them based on unearthing facts about them. This search engine employs thousands of individuals mobilized with one aim, to dig out hidden facts and expose individuals. The practice of the renrou search was firstly used in 2001 and then became a 'buzzword' in 2008, when there was a peak of renrou searching. Human-flesh search engines renrou sousuo yinqing - have become a Chinese phenomenon: they are a form of online vigilante justice in which Internet users hunt down and punish people who have attracted their wrath.

\section{Continuing State Regulation}

As Lagerkvist (2006) highlights, there is a central paradox in China's contemporary internet development: control and freedom seem to increase simultaneously. This is true of Weibo. One of the key distinguishing features of apublic sphere is its freedom from state interference; however, China's prevailing "Great Firewall” of internet filtering technology prevents the rise of an independent public sphere online. Despite rhetoric highlighting that the internet has brought some new freedoms, cyberspace, including Weibo, has never been free of administrative oversight. The internet is monitored and regulated by all governments to various degrees.

\subsection{Conventional Weibo Regulation}

Since the PRC's founding in 1949, China's Communist Party has taken information oversight as one of its primary governing strategies to ensure political stability. Since that time, all mass media, including newspapers, magazines, televisions and radio have been restricted by the authorities. This has also been true of the Internet since its introduction in 1987. The government has taken a series of measures to monitor online content and activities. These widely known techniques include surveillance, detention, propaganda or the "Great Firewall of China". It is at the same time making every effort to control Weibo - as it does with the internet generally.

Moreover, the party-state has adopted different technologies to monitor the medium. Not only is the regime engaged in public opinion channelling through its state-controlled media, but it also employs a handful of opinion commentators such as an entity known as the " 50 Cents Party" to try to actively shape the discussion of on the social media. The 50 Cents Party are online commentators said to be hired by the Chinese government (both central and local) or the Communist Party to post comments that are favourable towards party policies in an attempt to shape and sway public opinion on various internet message boards. They are said to receive 50 cents for each such post. 


\subsection{Beyond Censorship}

Apart from direct, "hard" censorship, which is an illiberal measure, the Chinese government has also adopted soft and subtle measures to become an active participant on Weibo. Facing the power of the social media, states including China have not prohibited the spread of new communication technologies; rather, they have taken some proactive attitude. Since the flourishing of social media, many countries have made use of it for various purposes. Weibo appeals to Chinese government in a number of ways: promoting transparency, fighting corruption and publicizing information.

Weibo has played increasingly important role in the field of providing information in emergencies, setting public issues, gathering social resources and generating public opinion. Within a short period, increasing number of government agencies and officials has opened Weibo accounts for interacting with citizens through this channel. The year of 2011 was labelled "the year of government Weibo" in China. Because of its efficiency and service, some officials' influence has been greatly improved. The utilization of government officials and department of Weibo is to make the governing process much convenient and to build a more effective bureaucracy and more transparent government.

Weibo has also been used to bring corrupt government officials to account. On 26th of August, 2012, the wrong doings of Yang Dacai - an official in north-western Shaanxi province appeared online. His behaviour sparked an outcry when pictures posted on Weibo showed him smiling while visiting a traffic accident site where 36 people had died. This was followed by images showing that he too was wearing a luxurious watch that critics considered he would not have been able to afford on his salary. Chinese internet users discussed his alleged corruption on Weibo and other online forums, requesting the local government dismiss Mr Yang, after which, he was sacked by provincial officials for "serious wrongdoing".

There is no doubt that Weibo will continue to be subject to increased official scrutiny while it also allows government to shape public opinion in subtle ways. Therefore, no complete public sphere could be formed under such circumstance as long as the authorities still apply electronic surveillance on Weibo.

\section{Conclusion}

In summary, Weibo is primarily a public space and does not serve as a platform for ideally deliberative discourses. Weibo has managed to create new public space for communication, however, greater participation in this space cannot guarantee a healthy and democratic public sphere, and it fails the Habermasian test. To enable a successful shift from public space to public sphere requires specific conditions which do not currently exist, such as a relative autonomy from the state and the economy. Nevertheless, with these official gestures in the direction of a public sphere, Weibo does provide a forum for the provision of two-way political communication, helping the government to access different voices and gain feedback, thus establishing a relationship between the government and the ordinary people. Different from the past visible censorship, strategies for media regulation in contemporary China are softer and more subtle than in the past. The party-state has enlarged the span of official tolerance of media freedom in some aspects, such as encouraging Weibo to monitor legal abuse, corruption and other wrongdoings outside the higher levels of the political system, but also in order to improve government accountability. Nevertheless, citizens' engagement in online protests indicates that public awareness of rights and accountabilities is evolving, although the extent to which it challenges injustice is limited. Only when the contradictions in China's current socio-economic conditions are significantly eased, when people's media literacy is much stronger and netizens become sufficiently informed to pay more attention to media ethics, will we be able to discuss the formation of a public sphere on Weibo and in social media more generally. In the meantime, China's party-state is acutely aware of the effectiveness of Weibo (and other social media) as a communication tool and is responding to public events in ways that suggest a more tolerant and positive tone toward a transformative public sphere. 


\section{References}

[1] Calhoun, C. (1992) Introduction. In C. Calhoun (ed.), Habermas and the public sphere (pp. 148). Cambridge: MIT.

[2] Castells, M. (2009) Communication power. Oxford: Oxford University Press.

[3] Fu, K. W., \& Chau, M. (2013) Reality Check for the Chinese Microblog Space: A Random Sampling Approach. PLoS ONE, 8(3): e58356. doi:10.1371/journal.pone.0058356

[4] Habermas, J. (1991) The structural transformation of the public sphere: An inquiry into a category of bourgeois society. Cambridge: MIT press.

[5] Habermas, J. (2009) Europe: The faltering project. Cambridge: Polity.

[6] Lagerkvist, J. (2006) The Internet in China: Unlocking and containing the public sphere.Lund: Lund University Press.

[7] Papacharissi, Z. (2002) The virtual sphere: The internet as a public sphere. New media \& society, 4(1), 9-27.

[8] Verstraeten, H (1996) 'The media and the transformation of the public sphere: a contribution for a critical political economy of the public sphere', European Journal of Communication, 11(3), 347370.

[9] Yang, G. (2009) The power of the Internet in China: Citizen activism online. New York: Columbia University Press.

[10] Zheng, Y. (2008) Technological empowerment: The Internet, state, and society in China. Stanford: Stanford University Press. 